\title{
Estudo e Aplicação da técnica Learning Mosaic no apoio à autorregulação da aprendizagem em Cursos Abertos Online e Massivos (MOOCs)
}

\author{
Aracele Garcia de Oliveira Fassbinder ${ }^{1,2}$, Ellen Francine Barbosa ${ }^{2}$ \\ ${ }^{1}$ Instituto Federal de Educação, Ciência e Tecnologia do Sul de Minas Gerais \\ IFSULDEMINAS Campus Muzambinho, Muzambinho - MG. \\ ${ }^{2}$ Instituto de Ciências Matemáticas e de Computação (ICMC) \\ Universidade de São Paulo (USP), São Carlos - SP. \\ aracele@ifsuldeminas.edu.br, francinedicmc.usp.br
}

\begin{abstract}
This paper presents a technique named Learning Mosaic that is generally used in enterprises to support self-regulation and personal and professional knowledge. Although its potential to support and stimulate selfregulated learning, Learning Mosaic is under explored in the context of teaching and learning, mainly in virtual courses. In additional, there is a demand by web tools to facilitate its application. This gap was identified during the delivering of a MOOC about Agile Software Development. The current results are useful for educators and mainly technologists interested in the development of MOOC platforms that support the related technique to enhance self-regulated learning in the open and virtual environments.
\end{abstract}

Resumo. Este artigo apresenta uma técnica denominada Learning Mosaic, geralmente utilizada no contexto presencial para apoiar a autorregulação da aprendizagem e gestão do conhecimento dentro de empresas. Apesar do potencial da técnica, ela ainda é pouco explorada no contexto de ensino e aprendizagem, principalmente em cursos virtuais, e existe uma demanda por ferramentas que automatizem sua aplicação. Essa questão foi identificada durante a oferta de um curso aberto online e massivo (MOOC) sobre Desenvolvimento Ágil de Software. Os resultados obtidos podem contribuir para aplicações futuras da técnica em ambientes presenciais ou virtuais e, também, estimular a criação ou adaptação de plataformas de MOOCs cujas funcionalidades sejam baseadas nos fundamentos de Learning Mosaic para fomentar a autorregulação da aprendizagem.

\section{Introdução}

A evolução das tecnologias computacionais e a disseminação da internet têm promovido redefinições na forma ensinar e aprender. Nesse contexto, a construção de conhecimento e o desenvolvimento de habilidades podem acontecer em qualquer espaço físico ou virtual, de maneira formal, informal ou não-formal.

De acordo com Inamorato dos Santos et al. (2016), por educação formal entendese aquela relacionada aos cursos formalmente reconhecidos por autoridades competentes, que seguem um conteúdo programático previamente definido, que normalmente exigem pré-requisitos ou processos seletivos para ingresso, e que o aluno está formalmente matriculado e receberá um certificado de conclusão caso seja aprovado. Já a educação 
não-formal pode envolver, de forma genérica, a aprendizagem por meio de estratégias menos restritivas, onde não há a obrigatoriedade de provas de ingresso, matrícula formal e/ou certificado oficial de conclusão. A educação informal, por sua vez, relaciona-se com o conhecimento adquirido por meio de experiências pessoais ou profissionais no cotidiano.

No contexto da educação não-formal, destacam-se os Cursos Abertos Online e Massivos, do inglês Massive Open Online Courses (MOOCs). O termo MOOC foi inicialmente utilizado em 2008, como uma forma de representar cursos virtuais que buscavam ampliar o acesso à educação para todos, por meio da remoção de barreiras, aprendizagem acessível e permitindo que diversas rotas de aprendizagem pudessem ocorrer de acordo com os objetivos dos estudantes (Fassbinder, Delamaro e Barbosa, 2014). O uso do termo vem evoluindo desde então. Em 2012, por exemplo, surgiram diversos provedores de MOOCs ligados às instituições de ensino e do mercado, principalmente americanas e europeias, tais como Coursera (https://www.coursera.org), edX (https://www.edx.org/), Udacity (udacity.com), MiríadaX (https://miriadax.net/home) e Future Learn (futurelearn.com). Esse contexto também tem estimulado o desenvolvimento de plataformas de código aberto, tais como open edX (open.edx.org), Google Course Builder (edu.google.com/openonline) e a representante brasileira Tim Tec (timtec.com.br/pt/funcionalidades). Com isso, qualquer pessoa ou instituição pode fazer o download, configurar e criar o seu próprio provedor de MOOCs.

Embora não exista um formato único e amplamente aceito para ofertar tais cursos, algumas características são recorrentes nos MOOCs:

- Qualquer pessoa pode acessar o ambiente virtual e se inscrever no curso.

- Alguns cursos definem uma data de início e fim do curso, mas durante esse período o aluno pode realizar as atividades na ordem e no tempo que desejaram.

- O foco está no apoio à aprendizagem ao longo da vida e no estimulo à autonomia do estudante em gerenciar ou regular a própria aprendizagem.

Apesar da popularidade dos MOOCs e das oportunidades de aprendizagem e socialização que eles podem proporcionar aos estudantes, os provedores e plataformas ainda são limitados sob o ponto de vista da incorporação de funcionalidades que apoiem o desenvolvimento de habilidades metacognitivas e, de forma específica, a autorregulação da aprendizagem pelos próprios aprendizes (Fassbinder, Fassbinder e Barbosa, 2016). Lima e Pimentel (2013), por exemplo, descrevem a arquitetura de uma ferramenta que incorpora mecanismos para propiciar a autorregulação da aprendizagem, mas trabalhos futuros incluem a sua incorporação em Ambientes Virtuais de Aprendizagem (AVAs), tais como Moodle e Sakai.

Considerando esse contexto, este trabalho teve como objetivo explorar a técnica de apoio à autorregulação da aprendizagem denominada Learning Mosaic, aplicando-a no contexto dos cursos abertos, online e massivos.

O artigo está organizado da seguinte maneira: a Seção 2 descreve os métodos utilizados no desenvolvimento do trabalho. A Seção 3 apresenta uma visão geral do funcionamento da técnica, seja em aplicação no ambiente presencial ou virtual; na Seção 4 é descrito um uso efetivo da técnica utilizando uma ferramenta da web 2.0 denominada Linoit.com; a Seção 5 descreve os resultados obtidos e alguns desafios também são pontuados, como forma de mostrar que ainda há muita dificuldade da utilização de 
estratégias plenas de apoio à autorregulação da aprendizagem, principalmente no contexto virtual e aberto; por fim, na Seção 6 são apresentadas as conclusões e na Seção 7 são apontados trabalhos de investigação que abordam a adaptação da técnica Learning Mosaic, bem como sua adaptação para o contexto dos MOOCs.

\section{Métodos}

A fim de compreender o funcionamento da técnica Learning Mosaic, iniciamos uma revisão de literatura, a fim de encontrar informações sobre a mesma. Identificamos que a técnica foi descrita e aprimorada por Magno (2015), em seu livro Learning 3.0. Magno (2015) é reconhecido, principalmente, pela comunidade de desenvolvimento ágil de software interessada em novas práticas de ensino e aprendizagem voltadas para incentivar o trabalhador criativo e empreendedor.

Em paralelo, um MOOC sobre desenvolvimento de software foi planejado. A fim de validar no contexto dos MOOCs a técnica já utilizada em uma oficina presencial de práticas ágeis que inspirou o MOOC em questão, iniciamos a identificação de ferramentas web que poderiam apoiar o uso da técnica, uma vez que a plataforma em que o MOOC foi ofertado não possuía suporte para a mesma. Dessa forma, a ferramenta linoit.com (http://en.linoit.com/) foi identificada e aplicada. Impressões dos estudantes sobre a técnica e seu uso no curso, a fim de apoiar a autorregulação da aprendizagem, foram identificadas por meio de questões inseridas em um questionário de autoavaliação.

\section{Learning Mosaic}

Nesta seção, são descritos o funcionamento da técnica denominada Learning Mosaic e os conceitos nos quais ela está embasada.

\subsection{Definição e Funcionamento}

De acordo com Magno (2015), Learning Mosaic é uma ferramenta que facilita a visualização do conhecimento e da aprendizagem de um jeito simples, mas adaptativo. Dessa forma, o indivíduo constrói, de forma contínua, um mosaico que reflete e expõe o conhecimento que emerge no contexto, seja no trabalho, na escola, ou durante um curso de qualificação profissional.

Inicialmente, cada pessoa constrói seu mosaico, cujas partes podem ser representadas por post-its ${ }^{1}$ coloridos. Inicia-se com o centro do mosaico, inserindo alguma figura ou avatar que a represente.

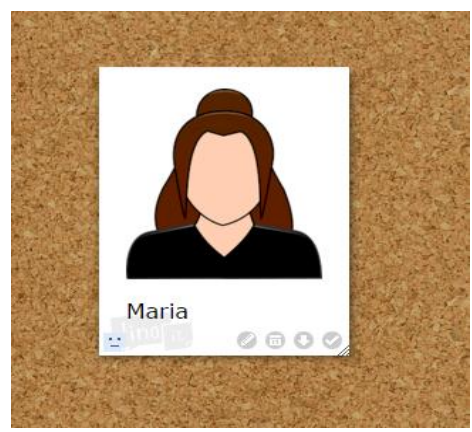

Figura 1 - Primeira etapa: identificação visual do estudante.

\footnotetext{
1 Pequeno papel com um adesivo de fácil remoção em seu verso, de forma que seja facilmente fixado, retirado e recolocado por algumas vezes.
} 
VI Congresso Brasileiro de Informática na Educação (CBIE 2017)

Anais do XXIII Workshop de Informática na Escola (WIE 2017)

Ao redor dessa identificação, devem ser colocados post-its que representam áreas nas quais ela gostaria de aprender ou reforçar novos conteúdos, bem como habilidades a serem desenvolvidas ao longo de um período, durante seu tempo em um curso, oficina, aula ou o próprio trabalho.

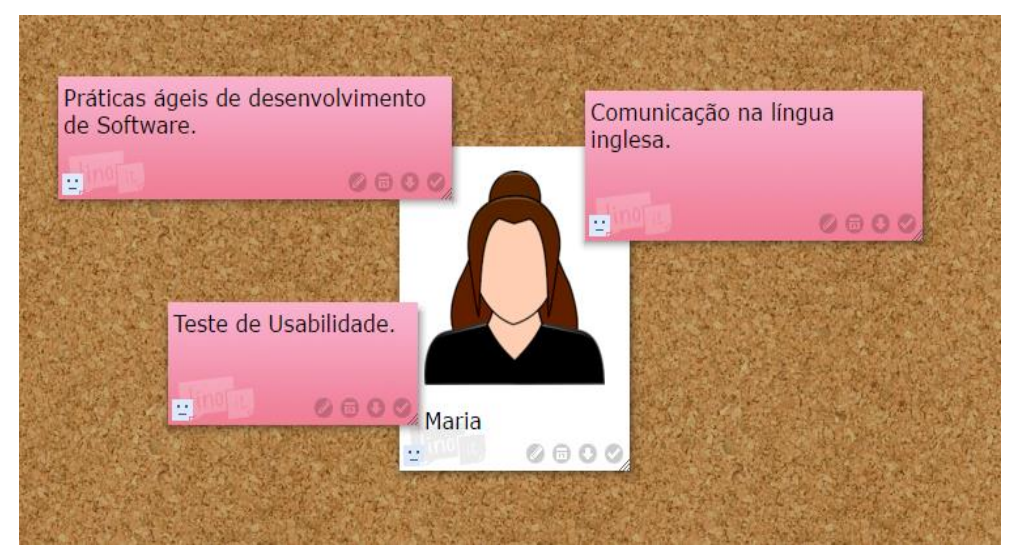

Figura 2 - Etapa 2: Inserção das áreas de interesse.

A partir desse momento, o mosaico deve ser periodicamente acessado e atualizado com a inserção de novos post-its que representam o que a pessoa aprendeu nas áreas inicialmente inseridas e dentro daquele determinado período.

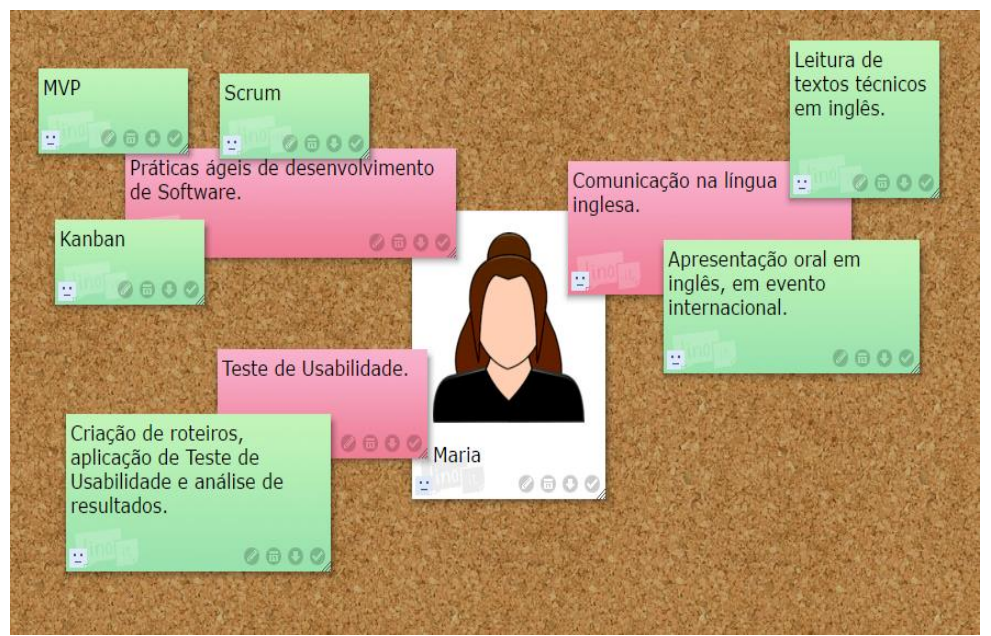

Figura 3 - Inserção dos conteúdos aprendidos elou habilidades desenvolvidas ou aperfeiçoadas, considerando as áreas inicialmente definidas.

Pode-se, também, inserir informações adicionais, tais como habilidades ou conteúdos não previstos, mas que foram desenvolvidos ou aprendidos. Outra possibilidade é registrar como isso ocorreu, ou seja, de que forma o conhecimento foi adquirido, por exemplo por meio de estudo em grupo, realização de experimentos, curso de extensão adicional, palestra, dentre outras. Com isso, o indivíduo consegue perceber o quanto ele aprendeu desde o início da construção do mosaico e como está a evolução da sua aprendizagem.

Adicionalmente, é importante manter os mosaicos por um certo período de tempo (durante todo o curso, toda a aula, oficina, etc.) pois, como eles ficarão disponíveis para todos, também é possível aprender com os demais, identificando a evolução de cada um e quanto conhecimento foi construído dentro de um curso, por exemplo. 
VI Congresso Brasileiro de Informática na Educação (CBIE 2017)

Anais do XXIII Workshop de Informática na Escola (WIE 2017)

Magno (2015) também enfatiza que novas regras ou características que adaptem a ideia original da técnica de Learning Mosaic podem ser realizadas dentro de cada contexto.

\subsection{Embasamento teórico e prático}

A técnica foi construída a partir da integração de alguns conceitos e práticas, conforme descrito nos próximos parágrafos.

Inicialmente, ela foi baseada em alguns princípios do conceito de Learning 3.0, também descrito em Magno (2015):

- Visualizar o conhecimento é mais importante do que medi-lo ou avaliá-lo;

- Compartilhar a própria aprendizagem cria novas oportunidades de aprender para quem compartilha e também para quem tem acesso ao conteúdo compartilhado.

- Pensar nas motivações e nos valores que a aprendizagem de um determinado conteúdo ou o desenvolvimento de uma habilidade pode acrescentar pessoal e profissionalmente, logo no início do curso, influenciará positivamente a qualidade do processo de aquisição de conhecimento e na significância das experiências vividas.

Também possui interseções com a área de Gestão de Conhecimento Pessoal, do inglês Personal Knowledge Management (PKM), que consiste de uma coleção de processos em que uma pessoa pode organizar, ordenar, armazenar, procurar, recuperar e compartilhar conhecimento sobre o seu dia-a-dia profissional, mas que também pode ser aplicado à vida pessoal (Razmerita, Kirchner e Sudzina, 2009). Alguns conceitos de Visual Thinking Ware (2010) também foram adicionados. E, por fim, tudo isso foi conectado a uma ferramenta pedagógica chamada Mosaico, utilizada pela Escola Lumiar, idealizada por Ricardo Semler (Lumiar, 2012). Na Lumiar, essa ferramenta possibilita a interação entre projetos que os alunos participam, a grade de conteúdo e habilidades curriculares, e o portfólio de aprendizagem de cada aluno.

\subsection{Validação inicial}

De acordo com Magno (2015), a ferramenta foi inicialmente validada no contexto de uma equipe de onze pessoas, as quais trabalhavam com campanhas de marketing em uma agência publicitária, em Nova York, Estados Unidos.

Isso possibilitou a elas:

- reflexão sobre o quê e como aprende;

- criação de um relacionamento e vínculo maior entre o trabalho e a aprendizagem;

- automotivação criada a partir da visualização do progresso diário do trabalho feito e, principalmente, das atividades desenvolvidas;

- compartilhamento de aprendizado com os demais membros;

- desenvolvimento de habilidades técnicas e não-técnicas.

\section{Aplicação no contexto dos Cursos Abertos Online e Massivos}

Embora a técnica de Learning Mosaic tenha sido aplicada no contexto presencial, conforme descrito anteriormente, nesta seção descrevemos seu uso no contexto de um MOOC denominado Desenvolvimento Ágil de Software. O MOOC foi ofertado nos 
VI Congresso Brasileiro de Informática na Educação (CBIE 2017)

Anais do XXIII Workshop de Informática na Escola (WIE 2017)

meses de Fevereiro e Março de 2017, e encontra-se disponível na instância da plataforma de MOOCs Tim Tec, no servidor do IFSULDEMINAS (https://mooc.ifsuldeminas.edu.br).

Como a versão atual (v4.1) da plataforma Tim Tec não possui funcionalidades de apoio à autorregulação da aprendizagem, seja por meio da técnica de Learning Mosaic ou outro recurso digital, fizemos uso de uma ferramenta web chamada linoit.com, criada para apoiar a postagem de notas adesivas virtuais. As notas adesivas, nessa ferramenta web, podem ser editadas, excluídas, rotacionadas, fixadas (para que não sejam movidas), dentre outros recursos.

A primeira semana do curso foi reservada para que os estudantes, orientados por meio de um vídeo e instruções textuais, refletissem sobre o conhecimento inicial que eles possuíam sobre práticas ágeis de desenvolvimento de software, seus conceitos e o contexto no qual estão inseridas, bem como os objetivos de aprendizagem que eles gostariam de alcançar ao final do MOOC. Eles também deveriam identificar estratégias pessoas para alcançar tais objetivos, tais como gerenciar melhor o tempo e criar um roteiro semanal de estudos. O objetivo dessa atividade foi despertar neles um amadurecimento e um comprometimento maior, enquanto estivessem realizando o curso. Para isso, os estudantes tiveram que acessar a linoit.com e criar seus mosaicos, utilizando as instruções:

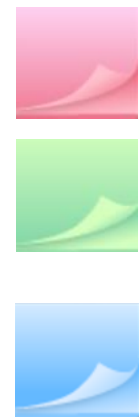

Novas adesivas ROSAS para coisas que você quer aprender ou desenvolver ao longo do curso.

Quando você sentir que aprendeu algo que tinha planejado, faça o login na ferramenta, vá até a nota adesiva relacionada, clique em editar e troque a cor para VERDE.

Caso perceba que aprendeu ou desenvolveu alguma habilidade que não tinha sido planejada, insira isso em uma nota adesiva de cor AZUL

Ao longo do curso, vídeos e mensagens estimulavam os aprendizes a manterem seus mosaicos sempre atualizados. E, ao final da quinta e última semana do curso, eles foram instruídos a realizar a última atualização do mosaico e preencher um questionário de autoavaliação da própria aprendizagem.

\section{Resultados e Discussão}

Cerca de 600 estudantes participaram do MOOC em questão. Neste trabalho, o foco está no uso da técnica Learning Mosaic facilitado pela ferramenta web linoit.com. Esse apoio à autorregulação da aprendizagem foi validado por meio de questões específicas inseridas em um questionário de autoavaliação aplicado aos estudantes que realmente finalizaram o MOOC, aproximadamente $12 \%$ do total de inscritos.

A Figura 4 apresenta um dos canvas criados na ferramenta linoit.com. Considerando o contexto massivo do curso, cerca de quatro canvas foram criados, a fim de receber os mosaicos dos estudantes envolvidos no curso de Desenvolvimento Ágil de Software. Cerca de $8 \%$ dos estudantes inscritos no curso participaram dessa tarefa. 
VI Congresso Brasileiro de Informática na Educação (CBIE 2017)

Anais do XXIII Workshop de Informática na Escola (WIE 2017)

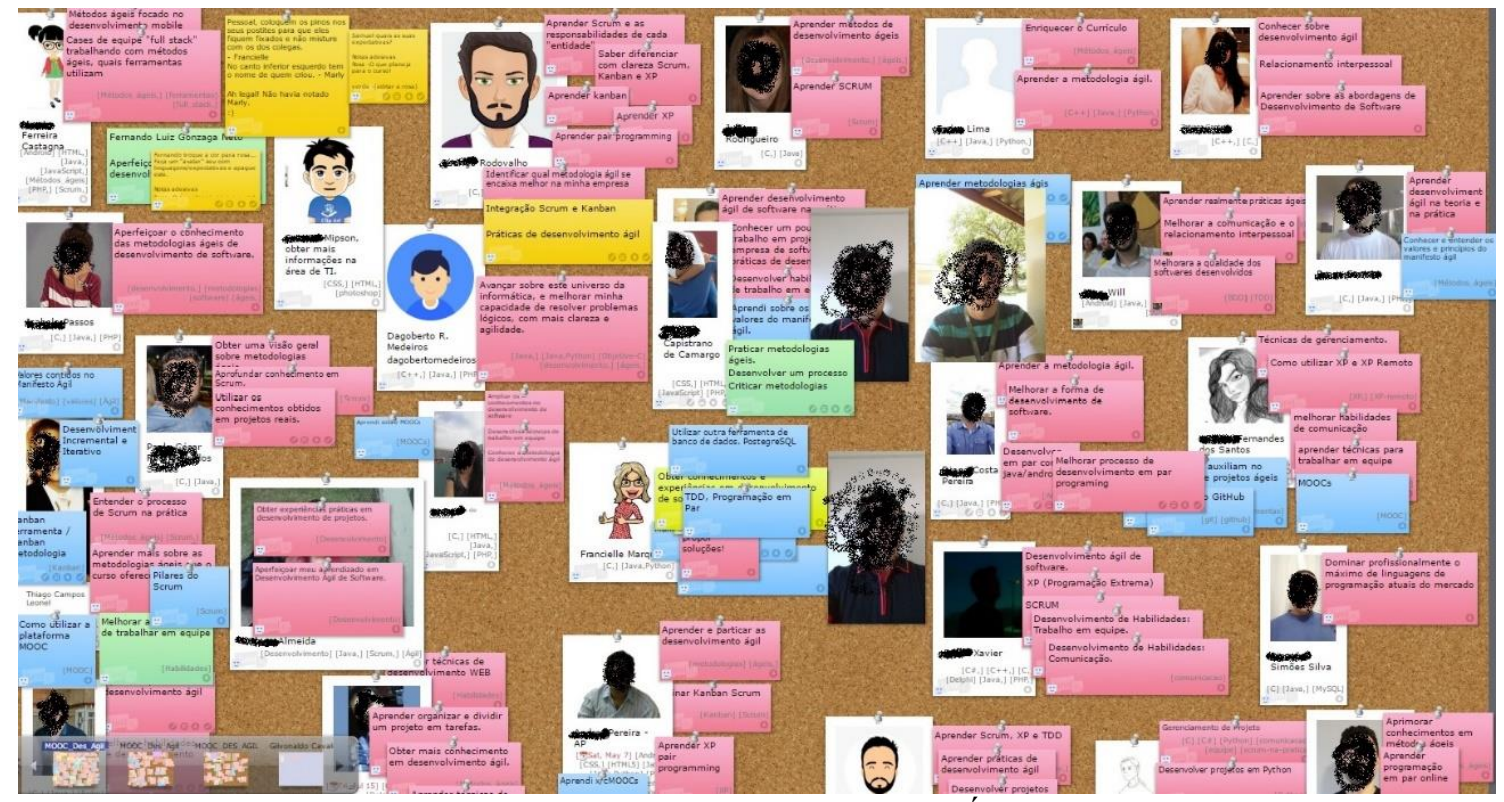

Figura 4 - Um exemplo de Canvas do MOOC de Desenvolvimento Ágil de Software na ferramenta Linoit.

As Figuras 5 e 6 apresentam a visão geral das respostas quando perguntados sobre a motivação em usar Learning Mosaic. Vale destacar que os estudantes envolvidos desconheciam a técnica e a sua finalidade. Sendo assim, além do conteúdo técnico relacionado ao desenvolvimento ágil, o uso da técnica trouxe novas perspectivas para os estudantes e sugestões de ideias futuras para gerenciar a própria aprendizagem ao longo da vida.

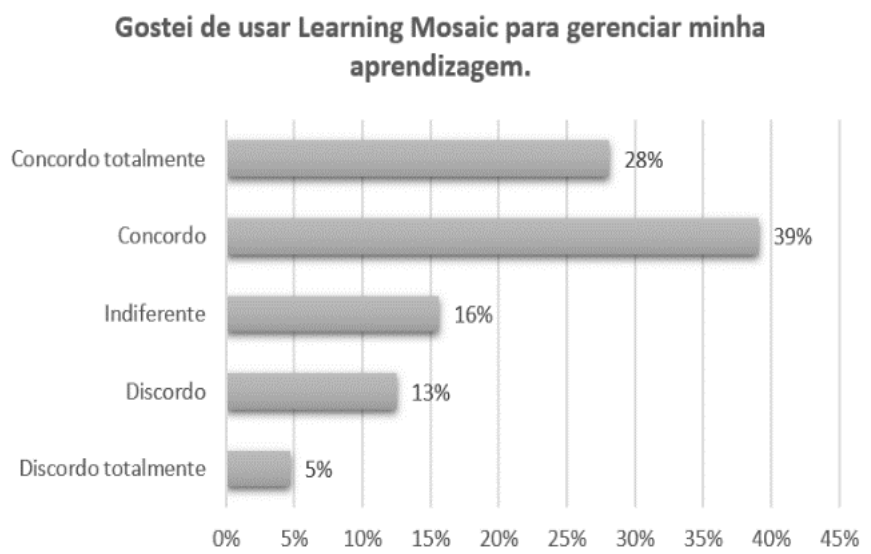

Figura 5 - Distribuição das respostas para a questão "Gostei de usar a técnica de Learning Mosaic para gerenciar minha aprendizagem”. 
VI Congresso Brasileiro de Informática na Educação (CBIE 2017)

Anais do XXIII Workshop de Informática na Escola (WIE 2017)

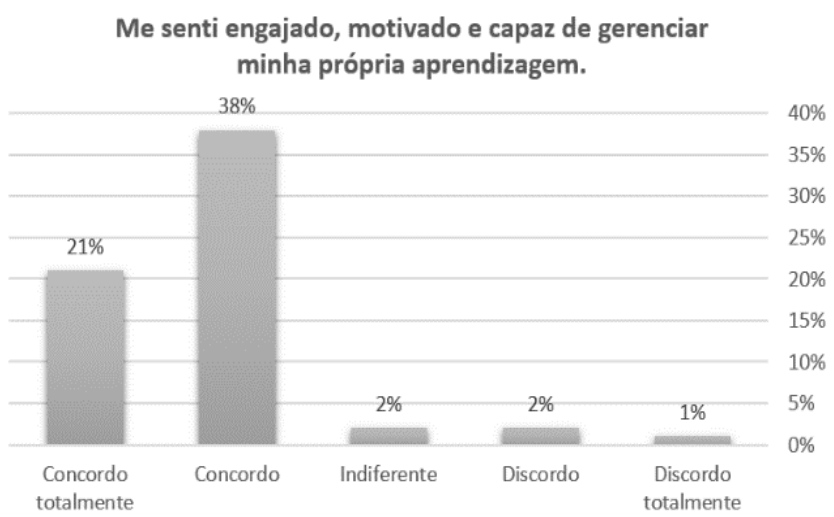

Figura 6 - Distribuição das respostas para a questão "Me senti engajado, motivado e capaz de gerenciar minha própria aprendizagem”.

O curso MOOC em questão teve uma data para início e fim das atividades pelos estudantes. Entretanto, o percurso dentro do curso poderia ser definido de forma livre, de acordo com as necessidades e interesses dos próprios estudantes. Adicionalmente, o período de inscrição no curso não foi encerrado. Assim, todos estavam aptos a se inscreverem e realizarem o curso, embora o número de desistência dos estudantes que se inscreveram entre a terceira e a quinta/última semana tenha sido maior. Essas características do curso podem ter influenciado nas respostas negativas apresentadas nas Figuras 7 (indiferente, discordo, discordo totalmente) e 8 (nula e baixa).

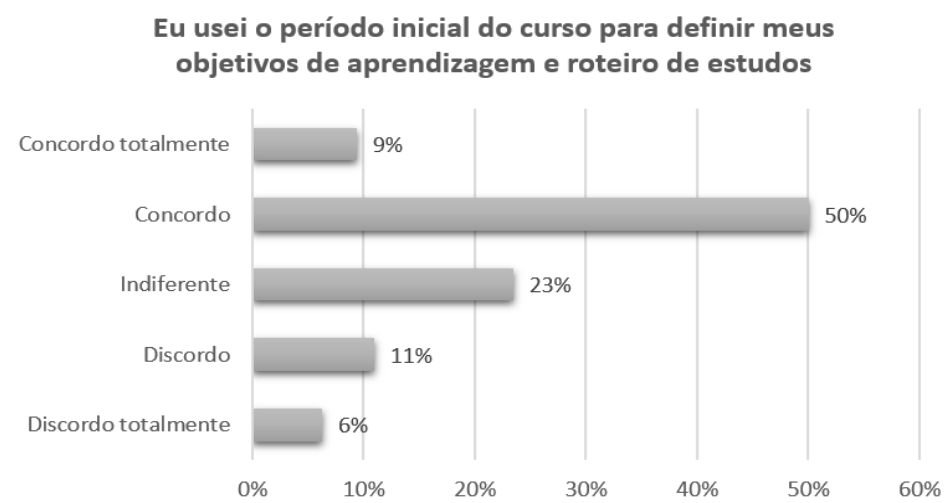

Figura 7 - Distribuição das respostas para a questão "Eu usei o período inicial do curso para definir meus objetivos de aprendizagem e roteiro de estudos".

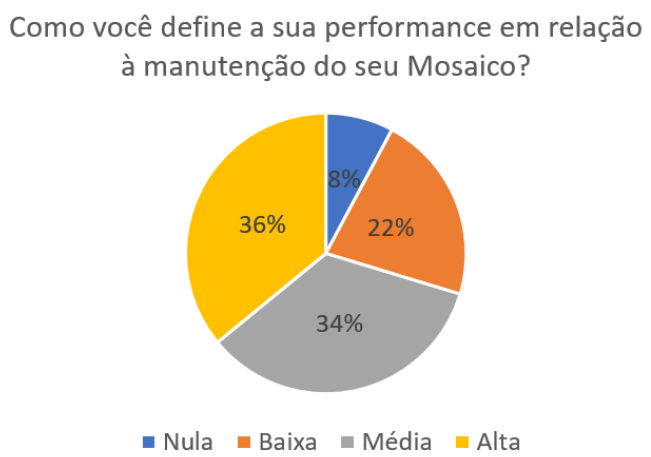

Figura 8-Distribuição de respostas para a questão "Como você define a sua performance em relação à manutenção do seu mosaico”? 
VI Congresso Brasileiro de Informática na Educação (CBIE 2017)

Anais do XXIII Workshop de Informática na Escola (WIE 2017)

Embora os resultados obtidos tenham sido positivos, é importante destacar que a ferramenta linoit.com não foi construída para atender a técnica Learning Mosaic. Algumas lacunas identificadas são:

- Credenciais de acesso duplicadas (um na plataforma do MOOC e outro na ferramenta linoit).

- A ferramenta está em inglês, que não é a língua nativa do curso e dos alunos inscritos.

- A área disponível para inclusão dos mosaicos (canvas) não suporta um número massivo de participantes.

- Não permite que o docente tenha uma visão sumarizada dos itens inseridos pelos estudantes, entre outras questões.

\section{Conclusão}

Este trabalho teve como objetivo apresentar o potencial da técnica Learning Mosaic como apoio à autorregulação da aprendizagem e a sua sistematização por meio da ferramenta Lino It. A aplicação da técnica e uso da ferramenta descrita foram exemplificadas por meio de um cenário de uso no contexto de um curso aberto e online sobre Desenvolvimento Ágil de Software.

Considerando o potencial da técnica estudada e as lacunas existentes na ferramenta utilizada neste trabalho, a linoit.com, investigações futuras envolvem o desenvolvimento de um software livre educacional, no formato de um plugin, que apoie a aplicação automatizada da técnica de Learning Mosaic, principalmente no contexto das plataformas abertas de cursos no formato MOOC.

\section{Agradecimentos}

As autoras agradecem o suporte financeiro das agências brasileiras de fomento (CAPES, Fapesp, Fapemig e CNPq) e do Instituto Federal de Educação, Ciência e Tecnologia do Sul de Minas Gerais - IFSULDEMINAS. Agradecimentos especiais ao Matheus Haddad, idealizador do Ateliê de Software da Webgoal, por meio do qual tivemos o contato com a técnica Learning Mosaic Tool.

\section{Referências}

Fassbinder, A., Delamaro, M. E. e Barbosa, E. F. (2014). "Construção e Uso de MOOCs: Uma Revisão Sistemática”, Simpósio Brasileiro de Informática na Educação-SBIE, Vol. 25, pp. 332.

Fassbinder, A. G. O., Fassbinder, M., Barbosa, E. F. (2016) "Um Conjunto Preliminar de Requisitos Pedagógicos para Caracterização e Comparação de Plataformas de MOOCs", In: XXI Congresso Internacional de Tecnologia Educativa (TISE), Santiago, Chile, Novembro.

Inamorato dos Santos, A., Yves, P. and Munoz, J. C. (2016) Opening up Education: A Support Framework for Higher Education Institutions. Publications Office of the European Union. Disponível em http://publications.jrc.ec.europa.eu/repository/handle/JRC101436. Acesso em 28 jun. 2017. 
VI Congresso Brasileiro de Informática na Educação (CBIE 2017)

Anais do XXIII Workshop de Informática na Escola (WIE 2017)

Júnior, J. C. M. da S., França, R. S. e Tedesco, P. C. de A. R. (2014) “AutoReg: uma ferramenta de apoio à autorregulação da aprendizagem", $3^{\circ}$ Congresso Brasileiro de Informática na Educação (CBIE), $20^{\circ}$ Workshop de Informática na Escola (WIE).

Lima, A. F. O. e Pimentel, E. P. (2013) "Mecanismos para Suporte à Auto-Regulação da Aprendizagem do Estudante", In Brazilian Symposium on Computers in Education (Simpósio Brasileiro de Informática na Educação-SBIE) (Vol. 24, No. 1, p. 296).

Lumiar, Escolas. (2012) “Como fazemos”. Disponível em lumiar.org.br. Acesso em 30 jun. 2016. http://lumiar.org.br/index.php/como-fazemos/

Magno, A. (2015) "How Creative Workers Learn: Develop your career with emergent learning and succeed in the creativity age." 1. ed. Happy Melly Express 180p.

Razmerita, L., Kirchner, K., Sudzina, F. (2009) "Personal knowledge management: The role of Web 2.0 tools for managing knowledge at individual and organizational levels", Online information review, v. 33, n. 6, p. 1021-1039, 2009.

Ware, C. (2010) "Visual Thinking for Design", San Francisco: Morgan Kaufmann Publishers. 